\title{
Theoretic Research on SMA External Pre-stress Concrete Structure
}

\author{
Zhigang $\mathrm{Yu}^{1, \mathrm{a}^{*}}$ and Rongrong Chen ${ }^{2, \mathrm{~b}}$
}

\author{
${ }^{1}$ School of Architecture Engineering, Chongqing Vocational Institute of Engineering, \\ Chongqing 402260, China \\ ${ }^{2}$ The Management Department, Chongqing Telecommunication Polytechnic College, \\ Chongqing 402247, China \\ ayzg3@sina.com, bcr3@@sina.com \\ *The corresponding author
}

\begin{abstract}
The effect of the external pre-stress on the mechanical properties of concrete structure was analyzed, on the basis of the shape memory effect, a new-style pre-stress structure on SMA external pre-stress concrete structure was put forward, the mechanical properties of ordinary reinforced concrete structure and SMA external pre-stress concrete structure have been analyzed comparatively, it is also pointed out in the paper that some problems should be solved before the structure are popularized and applied, and proposed the theoretical bases for engineering application of the external pre-stress structure of SMA for the future, the theoretical analysis show that the actuating effects of the shape memory alloy wires on the deformation of concrete structure can be obtained during phase transformation, the shape memory alloy actuator is successfully utilized as a way to control the crack width of concrete structure, the pre-stress structure can increase the crack resistance and the application of it is feasible.
\end{abstract}

Keywords: Shape memory alloy; External pre-stress; Concrete structure; Intelligence material; Reinforcement technology.

\section{形状记忆合金体外预应力混凝土结构理论研究}

\author{
余志刚 ${ }^{1}$ ，陈荣荣 ${ }^{2}$
}

（1.重庆工程职业技术学院建筑工程学院，重庆 400037；2.重庆电讯职业学院管理系，重庆 402247 )

摘要: 通过分析体外预应力对混凝土结构力学性能的影响, 基于形状记忆合金的形状记忆效应, 提出了一种新型的预应 力结构一形状记忆合金体外预应力混凝土结构, 对比分析了普通钢筋混凝土结构与形状记忆合金体外预应力混凝土结构的受 力性能, 指出了该结构在应用推广之前必须解决的问题, 为将来形状记忆合金体外预应力结构的工程应用提供了理论依据, 理论分析结果表明: 合金在相变过程中能对混凝土结构变形产生驱动效应，使得对混凝土结构裂纹宽度值的主动控制与调整 成为可能, 此种预应力结构能够提高抗裂能力且应用是可行的。

关键词: 形状记忆合金; 体外预应力；混凝土结构；智能材料；加固技术

中图分类号: TU378 文献标识码: A

引言

形状记忆合金（Shape Memory Alloy，简称 SMA）是近几十年发展起来的一种新型功能材料。这种材 料最主要的特征是具有形状记忆效应。形状记忆效应是美国哥伦比亚大学的 L. C. Chang 和 T. A. Read1951 年在 $\mathrm{AuCd}$ 合金中最早观察到的, 但一直到 60 年代初, 这只被看作是个别材料的特殊现象, 1963 年美国海 军军械研究室 W. J. Buehler 博士研究小组在 NiTi 合金中偶然发现具有实用价值的形状记忆效应, 形状记 忆合金才作为一种新型功能材料被人们所认识, 并成为一个独立的学科分支。经过 40 多年的研究和开发, 形状记忆合金已经进入商品化阶段, 1999 年, 我国的年销售量仅有 1 吨多, 到 2000 年就达到 4 吨以上, 
2001 年又增长到 6 吨左右, 2002 年全年的销售量则接近 10 吨。据估计, 近几年 TiNi 合金及其制品的销 售量应保持在 $25 \%$ 至 $30 \%$ 的增长速度 ${ }^{[1]}$ 。

所谓形状记忆效应（SME）, 是指材料会记住它在高温奥氏体态下的形状, 即它在低温马氏体态下变形, 加热后就会恢复到原来高温奥氏体状态下的形状 ${ }^{[2,3]}$ 。马氏体相变是SMA产生形状记忆效应的主要机制, 材 料内部的马氏体一旦生成, 会随温度的降低而生长, 随温度的升高而减少, 而相变的驱动力主要来源于两 相自由能之差。当温度低于平均温度 (两相自由能相等的温度) 时, 发生马氏体相变 (称为正相变); 当 温度高于平均温度, 发生奥氏体相变 (称为逆相变)。从形状记忆效应机理上看, 大部分合金和陶瓷等记 忆材料的形状记忆效应是通过马氏体相变而完成的, 也就是热弹性马氏体相变产生的低温相在加热时向高 温相进行可逆转变的结果, 但这种转变是有条件的, 以合金为例, 其条件必须是: 马氏体相变是热弹性的; 母体与马氏体相呈现有序点阵结构; 马氏体内部是孪晶变形的; 相变时在晶体学上具有完全可逆性 ${ }^{[4,5]}$ 。一 般合金的马氏体相变点与逆相变点是重合的，不具有形状记忆效应。

SMA在低温马氏体状态下变形，加热后残余变形消失，合金恢复到变形前奥氏体状态的形状即可逆应 力应变效应，当合金处于自由态时，该效应可用于对外输出应变; 当合金处于约束态时，则可对外输出应 力。本文主要分析了体外预应力对混凝土结构力学性能的影响，以形状记忆合金的形状记忆效应为基础, 提出一种新型的预应力结构-SMA体外预应力混凝土结构。

\section{SMA混凝土结构研究现状}

在柱体构件方面, 相关研究文献极少, 例如: 加拿大的Billah等 ${ }^{[6]}$ 对形状记忆合金和纤维增强多聚物 混合组成的混凝土圆柱进行了研究, 结果表明: 该柱体在振动期间, 耗能效果好, 减少了残余位移; 意大 利的Auricchio等 ${ }^{[7]}$ 通过挠度试验的模拟, 与试验结果进行了对比, 结果表明与试验结果吻合较好, SMA发 挥了较好的超弹性; 重庆交通大学韩西、等 ${ }^{[8]}$ 研究了形状记忆合金握裹在混凝土里的情况下其环向预应力 混凝土结构技术，结果表明SMA环向预应力混凝土柱与同等条件的普通混凝土柱相比，其抗裂性能有较大 的提高; 大连大学的崔迪等 ${ }^{[9]}$ 进行了形状记忆合金混凝土柱动力特性试验研究, 得出可以利用SMA来改变混 凝土构件或结构的动力特性，以有效控制结构振动。

在梁体构件方面, 相关研究文献较多, 例如: 美国Sreenath Kotamala ${ }^{[10]}$ 曾在混凝土梁中加入SMA, 结 果说明SMA在逆相变过程中能对梁产生很大的驱动力, 可以实现纵向预应力的施加; 清华大学的李磊等 ${ }^{[11]}$ 进行了基于形状记忆合金的智能混凝土梁桥设计与试验研究，结果表明：可以通过控制埋置在空心板内的 SMA束的温度控制其产生附加预应力的大小, 调节混凝土梁和桥的变形, 从而提高桥梁结构的承载能力。

\section{2 柱体结构}

\section{1 普通混凝土柱受力分析}

普通的混凝土柱在受到轴向压力作用以后, 随着压力的增大, 混凝土内部的微细裂缝不断发展, 横向 变形系数逐渐增大, 通常当轴向压应力达到 $0.5-0.7 f_{c}$ 界限值时, 微细裂缝开始急剧发展并相互之间迅速 贯通形成不可回逆的裂缝, 当压应力达到混凝土抗压强度时, 混凝土可能因折断而破坏, 也有可能因混凝 土内部形成的微小柱体失稳而引起整体失稳而破坏, 这种破坏一般不会引起粗骨料的压裂, 也就时说, 在 普通的混凝土柱中, 粗骨料不能很好发挥其良好的抗压性能。对于配有普通笔筋的轴向受压柱, 只有当混 凝土内部已经出现微细裂缝而引起体积横向膨胀挤压篃筋时, 才会使得䈐筋反过来约束混凝土横向变形, 
这时柱体才开始进入三向应力状态下工作。

\section{2 SMA混凝土柱体外预应力受力分析}

我们可以将SMA在常温下拉伸, 待混凝土柱浇注达到一定强度之后, 直接按需要的䈐筋间距缠绕在柱 体上, 在柱的两端针固好䈐筋, 再将SMA丝通稳压直流电, 待温度超过其相变点后, 它将产生恢复到原来 状态的趋势, 由于两端已经针固好, 这样材料内部产生的回复应力在混凝土柱体的阻挡下就实现了对结构 体外预应力的施加。

SMA体外预应力混凝土柱主要承受上部结构传递的轴向压力和体外预应力钢筋对混凝土横向变形产生 的预压应力两个力作用。对于普通的柱体构件, 利用约束混凝土概念, 如果提前对承受轴向压力的混凝土 柱上的SMA体外预应力筋通电使其产生形状记忆效应，使之使用之前就立即处于双向受压状态，以改善混 凝土受力性能, 则当柱一旦承受荷载开始工作时就立即处于三向受压的理想工作状态, 混凝土材料在三向 受压状态下, 各单元体的各棱边的总变形均比单向受力时要小, 其数学表达式为 ${ }^{[12]}$, 式中: $\varepsilon_{i}, \sigma_{i}$ 分别 为各向主应变和主应力 $(i=1,2,3) ; E, \mu$ 分别为弹性摸量和泊松比; 混凝土在三向受压作用下, 其 侧向压力越大, 限制或约束其横向变形的能力也越大, 所以其轴向所能承受的荷载值也越高, 此时顺着柱 纵向的微细裂缝的发展不但会较前者缓慢, 而且在较小的压力作用下, 这些微细裂缝还有重新闭合的趋势, 这样微细裂缝的发生和发展, 就只能当压力达到较高的情况下才会发生, 所以就使得混凝土的变形能力提 高, 只要侧向提供足够的侧压力, 柱因长期承载而形成的内部微小柱体始终不会失稳, 进而就能让混凝土 的破坏上升到更加高的一个级别, 让混凝土的粗骨料处于三轴压力之下, 使之在更加高的轴向压力下形成 更高一个级别的微小柱体, 直至这个级别的微小柱体因失稳而破坏, 在此受力状态下, 粗骨料充分发挥了 其本身良好的抗压性能, 使粗骨料的强度得到充分利用, 其结果表现应该是柱有良好的延性、柱的纵向承 载力得以提高。

$$
\begin{aligned}
& \varepsilon_{1}=\frac{\sigma_{1}}{E}-\mu\left(\frac{\sigma_{2}}{E}+\frac{\sigma_{3}}{E}\right) \\
& \varepsilon_{2}=\frac{\sigma_{2}}{E}-\mu\left(\frac{\sigma_{1}}{E}+\frac{\sigma_{3}}{E}\right) \\
& \varepsilon_{3}=\frac{\sigma_{3}}{E}-\mu\left(\frac{\sigma_{1}}{E}+\frac{\sigma_{2}}{E}\right)
\end{aligned}
$$

Table 1 The crack-resisting load and limit load of concrete columns

\begin{tabular}{cccc}
\hline $\begin{array}{c}\text { Column } \\
\text { number }\end{array}$ & $\begin{array}{c}\text { Crack-resisting load } \\
/ \mathrm{kN}\end{array}$ & $\begin{array}{c}\text { Limit } \\
\text { load } / \mathrm{kN}\end{array}$ & $\begin{array}{c}\text { Crack-resisting load / } \\
\text { Limit load }\end{array}$ \\
\hline 1 & 55.0 & 62.5 & 0.880 \\
2 & 55.0 & 60.5 & 0.909 \\
3 & 50.0 & 58.5 & 0.855 \\
Average & 53.3 & 60.5 & 0.881 \\
value & & & \\
\hline
\end{tabular}


Table 2 The crack-resisting load and limit load of bonded SMA concrete columns

\begin{tabular}{ccrc}
\hline $\begin{array}{c}\text { Column } \\
\text { number }\end{array}$ & $\begin{array}{c}\text { Crack-resisting load } \\
\text { /kN }\end{array}$ & $\begin{array}{c}\text { Limit } \\
\text { load } / \mathrm{kN}\end{array}$ & $\begin{array}{c}\text { Crack-resisting load / } \\
\text { Limit load }\end{array}$ \\
\hline 4 & 65.0 & 89.0 & 0.730 \\
5 & 60.0 & 77.0 & 0.779 \\
6 & 65.0 & 89.5 & 0.726 \\
Average & 63.3 & 85.2 & 0.745 \\
value & & & \\
\hline
\end{tabular}

Table 3 The crack-resisting load and limit load of unbonded SMA concrete columns

\begin{tabular}{ccrc}
\hline $\begin{array}{c}\text { Column } \\
\text { number }\end{array}$ & $\begin{array}{c}\text { Crack-resisting load } \\
\text { /kN }\end{array}$ & $\begin{array}{c}\text { Limit } \\
\text { load } / \mathrm{kN}\end{array}$ & $\begin{array}{c}\text { Crack-resisting load / } \\
\text { Limit load }\end{array}$ \\
\hline 7 & 55.0 & 88.5 & 0.621 \\
8 & 60.0 & 84.5 & 0.710 \\
9 & 50.0 & 79.0 & 0.633 \\
Average & 55.0 & 84.0 & 0.664 \\
value & & & \\
\hline
\end{tabular}

钟厉等 ${ }^{[13]}$ 研究了 NiTi 形状记忆合金体内环向预应力混凝土结构的强化现象, 试验结果如表 1 、表 2 和表 3, 试验结果表明 SMA 体内环向预应力混凝土柱与同等条件的普通混凝土柱相比, 其极限荷载和抗裂 性能都有较大的提高。理论上若对圆柱体外预应力筋分析, 则其比体内预应力筋在形状记忆效应发生时, 少了由于混凝土握裹的原因而产生的摩擦力效应带来的摩擦损失, 其预应力效果应该更明显。目前使用的 环向预应力技术需要以金属波纹管或塑料波纹管为预留孔道, 并需要采用特殊张拉端针具体系, 预留套筒 及配套张拉设备, 变角张拉等工艺, 但是造价较高、施工也很困难。SMA 加工性能相对良好, 可以进行冷 拉或者冷扎, 还具有性能稳定、强度和疲劳寿命高、耐腐蚀性好、与复合材料基本相融性好, 对应用中的 合金丝可以在其外层涂上防腐涂料以便保证其受长期工作环境的腐蚀。这种施加预应力的方法简便、快捷、 省力, 对于设计同样承载力要求的柱, 可以减小结构尺寸, 降低工程造价, 该技术不但可应用于所有轴向 受压构件, 尤其对于正在 “服役” 出现有微裂纹的柱体, 可以直接缠绕在柱体结构之外, 然后通电达到加 固的目的。

\section{3 梁体结构}

\section{1 普通混凝土梁受力分析}

第一阶段梁弯矩较小，这时的工作特点是梁尚未出现裂缝，在该阶段由于梁整个截面参加受力，截面 抗弯刚度较大, 梁的挠度很小, 且与弯矩近似成正比; 当弯矩超过开裂弯后, 在开裂瞬间, 裂缝截面受拉 区混凝土退出工作, 这时梁进入第二阶段, 其开裂前承担的拉力将转移给钢筋承担, 导致裂缝截面钢筋应 力突然增加, 使中和轴比开裂前有较大上移, 随着裂缝的出现与开展, 挠度的增长速度较开裂前为快, 荷 载继续增加, 挠度不断增大, 裂缝宽度也随荷载的增加而不断开展; 当受拉钢筋刚达到屈服强度时, 弯矩 达到屈服弯矩, 梁受力进入第三阶段, 其特点是梁的裂缝急剧开展, 挠度急剧增加, 而钢筋应变有较大的 
增长, 继续加载, 当受压区混凝土达到极限压应变时, 梁达到极限弯矩, 此时梁开始破坏。

\subsection{SMA 混凝土体外预应力梁受力分析}

如果在普通梁应用之前, 在其受拉区（如：槽形梁）体外应用SMA施加预应力, 理论上这种梁的受力 不但具有常用预应力钢筋混凝土梁的特点, 而且具有其特殊的智能功能：若利用SMA的单程记忆效应，一 旦梁出现类似普通混凝土梁第二阶段中的较大裂纹，可以随时对其通电，SMA将智能地发挥回复的应力可 达700 MPa，从而裂纹将产生重新闭合趋势。

该智能功能使得梁的使用寿命得到了延长，无需更换新的梁式结构，提高了经济效益，也为工程上梁 的加固提供了一条解决的途径, 特别对于桥梁中常用的大型梁式构件, 目前在全世界范围内加固工程方面 耗资巨大, 从长远利益来看, 如果使用SMA施加预应力, 只需通电加热使预应力筋产生应力, 不但省去了 通常施工中普通钢筋需要的复杂张拉过程, 而且也为该桥梁将来可能出现的加固问题提供了便利, 特别对 于由于空间上的不便而难以张拉钢筋的结构，既节省了工期，又保证了工程的质量。

\section{4 结语}

SMA材料是一种钢材，抗拉性能相当好，在理论上，SMA体外预应力关于计算方面问题的思路大体可以 按类似普通钢筋的相关计算过程来计算，目前为止，国内外关于SMA的体外预应力 “实际工程” 技术的研 究未见任何相关文献。为了使SMA体外预应力结构能够安全可靠地应用到实际的工程当中, 我们急需进行 大量的研究工作，解决与此相关的一系列问题：廉价高性能高温形状记忆合金的开发。如：铜基形状记忆 合金具有性能上的潜力、价格上的优势，铁基形状记忆合金由干价格低廉、强度高、加工性能好、使用方 便等优点; SMA的体外配筋计算的研究; SMA材料形状记忆特性、粘结条件剂尺寸效应等的深入研究; SMA 体外预应力工程技术的研究。

综合以上分析，一旦SMA体外预应力技术研究成熟，我们运用SMA体外预应力技术完成对结构的体外预 应力施加在理论上是完全可行的，该技术可应用于土木工程上柱体受压构件、梁式受弯构件及管状受内压 构件等需要体外预应力筋的结构, 我们可以用SMA代替普通的钢筋, 只需对其通电就能达到施加预应力的 目的，它将是一种应用前景非常好的功能材料。

\section{5 致谢}

\section{重庆市自然科学基金资助项目（CSTC05BB6116）.}

\section{Acknowledgment}

The research is supported by the Natural Science Foundation Projects of Chongqing CSTC (05BB6116).

\section{参考文献:}

[1] 杨冠军,杨华斌, 曹继敏. 我国形状记忆合金研究与应用的新进展 [J]. 材料导报, 2004, 18 (2) : 42-44.

[2] 杨杰, 吴月华. 形状记忆合金及应用[M]. 合肥:中国科技大学出版社, 1993.

[3] CHERNENKO V A, KOKORIN V V, VITENKO I N: "Properties of ribbon made from Shape Memory Alloy $\mathrm{Ni}_{2} \mathrm{MnGa}$ by quenching from the liquid state" [J]. Smart-Mater Struct, Vol. 3(1994), No. 1, p. 80-82.

[4] GANDHI M V, THOMPSON B S: "Smart Materials and Structures" [M]. London: Chapman \& Hall, 1992.

[5] 马超. 形状记忆材料的应用与发展 [J]. 辽宁化工, 2006, 35 (1) : 30-32.

[6] Billah M, Alam M S: "Seismic performance of concrete columns reinforced with hybrid shape memory alloy 
(SMA) and fiber reinforced polymer (FRP) bars" [J]. Construction and Building Materials, , Vol. 28(2012), No. 1, p. $730-742$.

[7] AURICCHIO F, SACCO E: “A Superelastic Shape Memory Alloy Beam Model” [J]. Journal of Intelligent Material Systems and Structures, Vol. 8(1997), No. 6, p. 489-501.

[8] 韩西, 余志刚, 钟厉. SMA环向预应力结构原理研究 [J]. 工业建筑, 2007, 37 (S1) : 325-327.

[9] 崔迪, 李宏男, 宋钢兵. 形状记忆合金混凝土柱动力特性试验研究 [J]. 振动与冲击, 2010, 29 (4): 150-154.

[10] Kotamala S: “Prestressing of simply supported concrete beam with Nitinol shape memory alloy" [D]. Ohio: University of Toledo, 2004.

[11] 李否, 李庆斌, 张帆. 基于形状记忆合金的智能混凝土梁桥设计与试验研究 $[J]$. 工程力学, 2010, 27 (3) :45-54.

[12] 蒋家奋, 汤关祚. 三向应力混凝土 [M]. 北京: 中国铁道出版社, 1988.

[13] 钟厉, 邓兰兰, 韩西, 余志刚, 杨明理. NiTi 形状记忆合金环向预应力混凝土结构强化现象的研究 [J]. 混凝 土, 2008, (1) :29-31.

\section{References}

[1] Yang Guan-Jun, Yang Hua-Bin, Cao Ji-Min: “ New advances in research and applications of Shape Memory Alloys in China"[J]. Materials Review, Vol. 18(2004), No.2, p.42-44 (in Chinese)

[2] YANG Jie,WU Yue-hua: Shape Memory Alloy and its application"[M].Hefei:University of Science and Technology of China Press, 1993. (in Chinese)

[3] CHERNENKO V A, KOKORIN V V, VITENKO I N: "Properties of ribbon made from Shape Memory Alloy Ni2MnGa by quenching from the liquid state"[J]. Smart-Mater Struct, Vol. 3(1994), No.1, p.80-82.

[4] GANDHI M V, THOMPSON B S: "Smart Materials and Structures" [M]. London: Chapman and Hall,1992.

[5] MA Chao: "Discussion on Shape Memory Materials"[J]. Liaoning Chemical Industruy, Vol. 35(2006), No.1, p.30-32 (in Chinese)

[6] Billah M, Alam M S: "Seismic performance of concrete columns reinforced with hybrid shape memory alloy (SMA) and fiber reinforced polymer (FRP) bars"[J]. Construction and Building Materials, , Vol. 28(2012), No.1, p.730-742.

[7] AURICCHIO F, SACCO E: "A Superelastic Shape Memory Alloy Beam Model"[J]. Journal of Intelligent Material Systems and Structures, Vol. 8(1997), No.6, p.489-501.

[8] HAN Xi, YU Zhi-gang, ZHONG Li: "Research on Structural Principle of Ring Pre-stress Based SMA"[J]. Industrial Construction, Vol. 37(2007), No.S1, p.325-327 (in Chinese)

[9] CUI Di, LI Hong-nan,SONG Gang-bing: "Experimental Study on Dynamic Properties of SMA Reinforced Concrete Columns"[J]. Journal of Vibration and Shock, Vol. 29(2010), No.3, p.150-154 (in Chinese)

[10]Kotamala S: "Prestressing of simply supported concrete beam with Nitinol shape memory alloy"[D].Ohio: University of Toledo,2004.

[11]LI Lei, LI Qingbin, ZHANG Fan: "Design and Experiment of a Smart Concrete Bridge with Embedded Shape Memory Alloy Bundles"[J]. Engineering Mechanics, Vol. 27(2010), No.3, p.45-54 (in Chinese)

[12]Jiang Jia-fen, Tang Guan-zuo: "Three direction stress concrete"[M]. Beijing: China Railway Publishing House, 1988. (in Chinese)

[13]ZHONG Li, DENG Lan-lan, HAN Xi, et al: "Study on the strengthening of NiTi SMA ring pre-stress concrete structure'[J]. Concret, (2008), No.1, p.29-31 (in Chinese)

[14] 